\title{
Creación laparoscópica de neovagina según técnica de Davydov modificado, en pacientes con síndrome de Rokitanski
}

\author{
Laparoscopic creation of a neovagina following modified Davydo's technique in patients with Rokitanski's \\ syndrome
}

\begin{abstract}
Adolfo Rechkemmer Prieto ${ }^{1, a, b}$
\end{abstract}
\section{RESUMEN}

Se describe una técnica quirúrgica alternativa para la formación de neovagina en ocho casos de agenesia de útero y vagina. Todas las pacientes tenían amenorrea primaria y ausencia congénita de útero y vagina (Síndrome de Rokitansky), y tenían entre 18 y 25 años de edad. De manera breve, la técnica consistió en hacer una disección roma en el espacio vésico-rectal hasta alcanzar el peritoneo. Se realizó tracción del peritoneo hacia abajo con una pinza, se abrió y se suturó con vicryl 0 al borde del orificio de la neovagina a nivel del introito. Por vía laparoscópica se cerró el peritoneo formando así la cúpula de la nueva vagina. Se colocó en la neovagina un molde de espuma de goma cubierto con un condón. La estancia hospitalaria osciló entre 2 y 5 días. No se presentaron complicaciones post operatorias. Luego de uno a seis meses el examen pélvico mostró una neovagina con una profundidad de 6 a 10 $\mathrm{cm}$, sin tejido de granulación. Se concluye que la construcción de una neovagina usando peritoneo pélvico asistido por laparoscopía, seguido de un molde vaginal de espuma de goma, es una técnica eficiente y segura.

PALABRAS CLAVE: Vagina, anomalías congénitas, laparoscopía. (Fuente: DeCS BIREME).

\section{SUMMARY}

We report an alternative surgical procedure for creating a neovagina in eight patients with agenesis of the uterus and vagina. All patients presented with primary amenorrhea and congenital absence of the uterus and vagina (Rokitanski's syndrome) and had 18-25 years of age. In brief, a dissection of the bladder-rectal space was performed until reaching the peritoneum that was subsequently pull down with a gripper to attach it to the orifice of the neovagina at the introitus using sutures with vicryl 0 . Then, by using laparoscopy, the peritoneum was closed creating the dome of the vagina. A mold of foam cover by a condom was inserted in the neovagina. Hospital stays ranged from 2-5 days, no post-operative complications were observed. Six months after, the pelvic examination showed a vagina of $6-10 \mathrm{~cm}$ in deep with no granulation tissue. We conclude that creating a neovagina using a laparoscopic assisted procedure followed by a foam mold is efficient and safe.

KEYWORDS: Vagina, congenital abnormalities, laparoscopy. (Source: MeSH NLM).

1. Facultad de Medicina, Universidad Peruana Cayetano Heredia. Lima, Perú.

a. Profesor Principal;

b. Doctor en Medicina 


\section{INTRODUCCIÓN}

El síndrome de Mayer-Rokitansky-Kuster-Hauser es una alteración congénita poco frecuente del aparato reproductor femenino, con una prevalencia de 1 en 5,000 nacimientos de sexo femenino ${ }^{(1)}$. Han sido descritas varias técnicas para el tratamiento de la ausencia de vagina en estas pacientes, incluyendo dilatadores vaginales (2), moldes cubiertos de piel ${ }^{(3)}$, injertos de sigmoides ${ }^{(4)}$, y colgajos de vulva y músculos ${ }^{(5)}$. En 1965, Vecchietti ${ }^{(6)}$ propuso un método que puede ser considerado una versión quirúrgica del método de Frank, aplicando una tracción constante hacia arriba, causando una profunda invaginación en el espacio recto-vaginal. Este método fue posteriormente modificado por vía laparoscópica ${ }^{(7)}$. Davydov publicó su experiencia con laparotomía y el uso de peritoneo para recubrir el espacio vésico-rectal recién disecado (8). Una característica de estas técnicas quirúrgicas es que requieren varios días de internamiento y es característico el dolor postoperatorio.

El objetivo del reporte fue presentar los resultados de un abordaje laparoscópico mínimamente invasivo del procedimiento de Davydov para la creación de una neovagina en casos de agenesia útero-vaginal.

\section{PRESENTACIÓN DE LOS CASOS}

Se presentan ocho pacientes con edades entre 18 y 25 años con historia de amenorrea primaria e imposibilidad para tener relaciones coitales, que acudieron entre enero de 2007 y marzo de 2015, seis de ellas atendidas en el Hospital Nacional Arzobispo Loayza de Lima, y dos en la práctica privada. El examen ginecológico mostró en todos los casos ausencia completa de vagina y caracteres sexuales secundarios normales. El ultrasonido pélvico mostró ausencia de útero. Los estudios radiológicos mostraron doble sistema pielo-calicial en un caso y riñón de localización pélvica en otro caso. Las otras seis pacientes no presentaron anormalidades renales. Ninguna presentó anomalías esqueléticas. El cariotipo en linfocitos de sangre periférica mostró un complemento cromosómico femenino normal.

\section{Descripción de la técnica quirúrgica}

Bajo anestesia general, se colocó a la paciente en posición de litotomía y se le realizó una laparoscopía pélvica, que demostró la presencia de ambos ovarios; en cinco pacientes se encontró cuerno uterino rudimentario no funcionante, $\mathrm{y}$ en las restantes

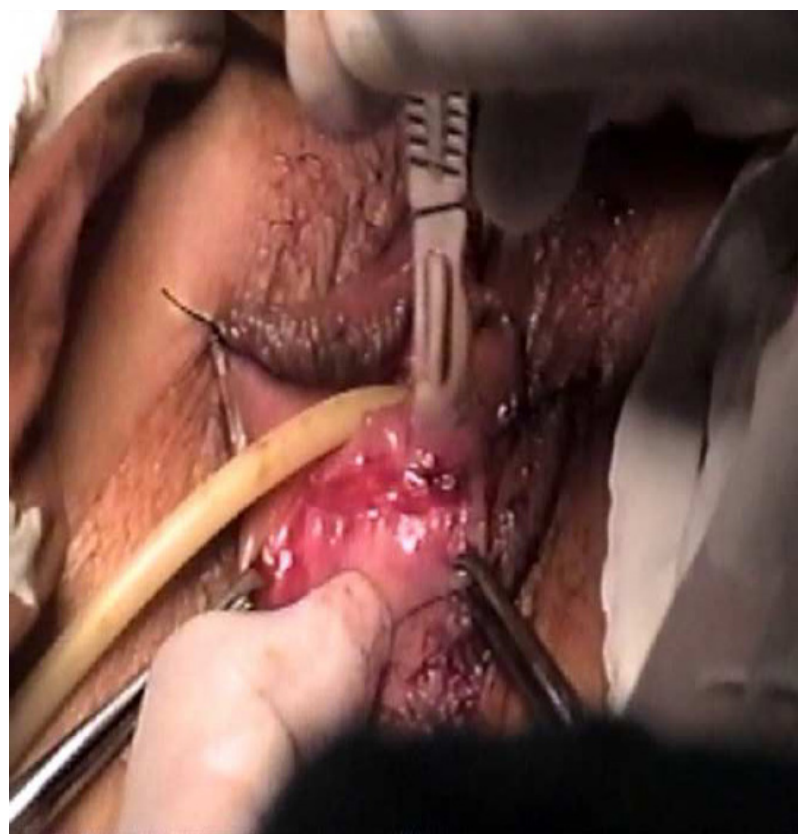

Figura 1. El examen del introito muestra ausencia completa de vagina. Incisión transversa en el introito y disección roma en el espacio vésico-rectal

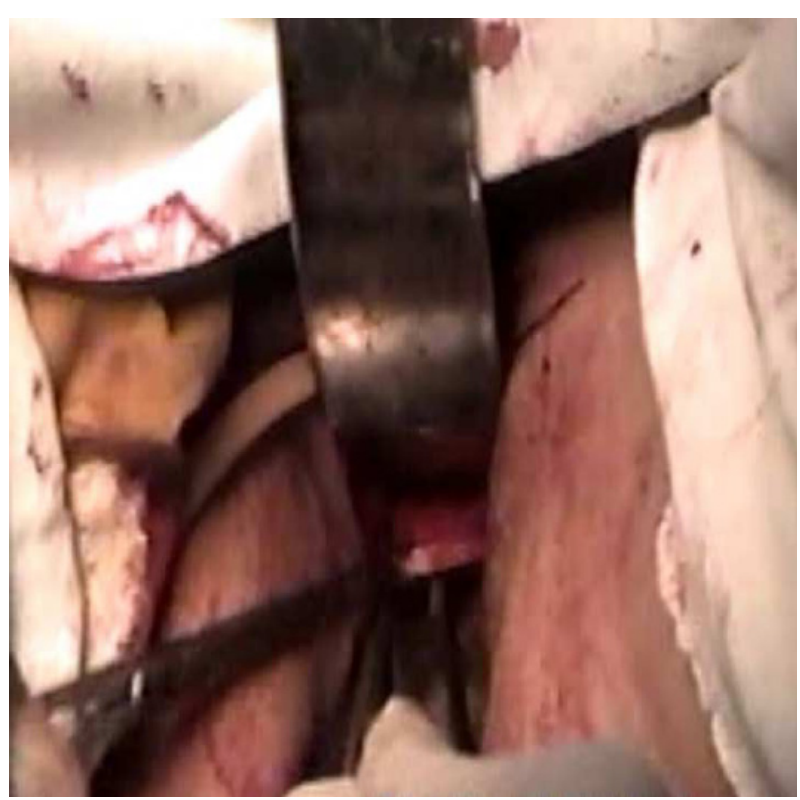

Figura 2. Se realiza tracción del peritoneo a través de la neovagina recién creada.

agenesia completa de útero. A nivel del introito se realizó una incisión horizontal (figura 1), y se realizó disección roma en el espacio vésico-rectal hasta alcanzar el peritoneo. Por vía laparoscópica se realizaron incisiones peritoneales por encima de la vejiga y las paredes laterales de la pelvis para relajar el peritoneo. Se introdujo una pinza de Allis a través de la neovagina recién creada y se pinzó y traccionó 


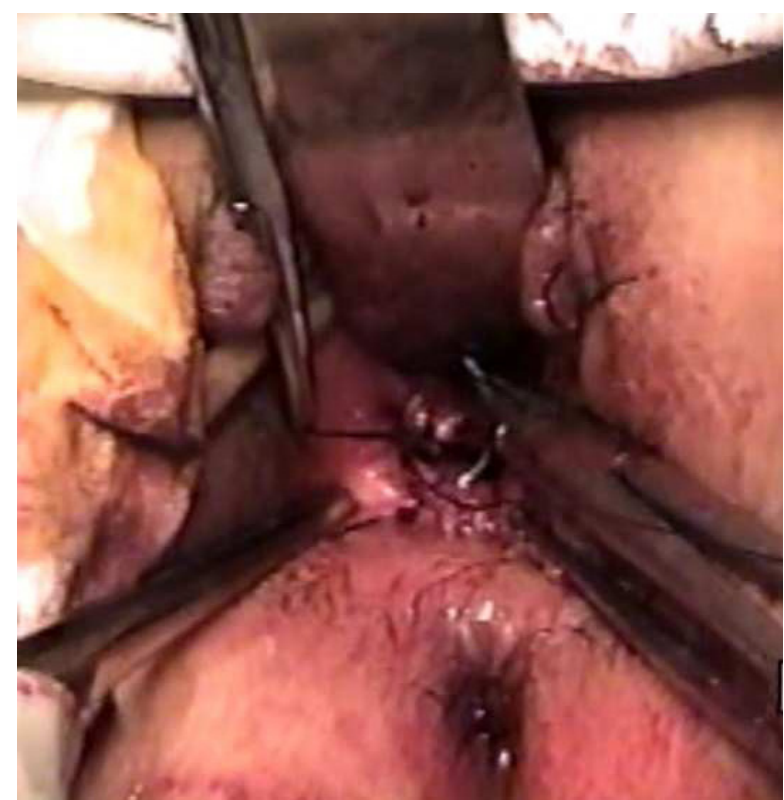

Figura 3. Se sutura el peritoneo al borde del orificio de la neovagina en el introito.

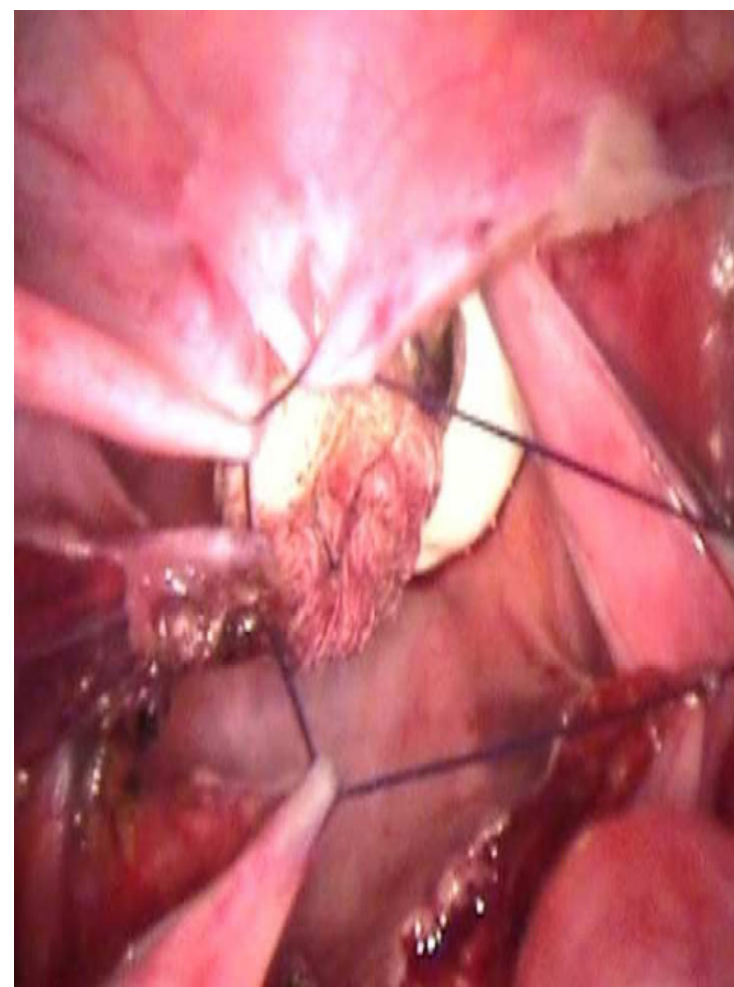

Figura 4. Por vía laparoscópica el peritoneo es suturado con vicryl 2/0 y cerrado en bolsa de tabaco.

el peritoneo hacia abajo (figura 2), se corta y fija el peritoneo con sutura vicryl 0 al borde del orificio de la neovagina a nivel del introito (figura 3). Luego de esta movilización el peritoneo recubre la neovagina. La cúpula de la vagina reconstruida se forma al aproximar el peritoneo por vía laparoscópica empleando sutura vicryl 2-0, cerrándola en "bolsa de tabaco" (figura 4). Al final se colocó dentro de la neovagina un molde vaginal temporal blando hecho con espuma de goma cubierto con un condón, el que fue retirado a las 48 a 72 horas.

Las pacientes fueron dadas de alta entre 2 y 5 días luego de la intervención quirúrgica, sin complicaciones mayores. Una paciente presentó edema leve en genitales externos, que cedió espontáneamente a los 30 días.

Se les pidió a las pacientes que usen el molde vaginal solamente en las noches. El examen ginecológico a los 30 días después de la cirugía mostró una neovagina de 6 a $10 \mathrm{~cm}$. de profundidad sin presencia de tejido de granulación. A partir del tercer mes luego de la cirugía, tras confirmar que la vagina se encontraba totalmente epitelizada, se les permitió tener relaciones coitales y suspender la inserción del molde vaginal. Seis pacientes tuvieron un control a los seis meses, mostrando evolución favorable y refiriendo relaciones sexuales satisfactorias.

\section{DISCUSIÓN}

Se presentan los resultados de un abordaje laparoscópico mínimamente invasivo del procedimiento de Davydov para la creación de una neovagina en casos de agenesia útero-vaginal. Este procedimiento permitió formar una vagina funcional en las pacientes tratadas, que les permitió tener una adecuada vida sexual.

La creación de una neovagina sexualmente funcionante debería originar mínimas molestias durante el periodo de evaluación diagnóstica, intervención quirúrgica y período post operatorio. Adamyan ${ }^{(9)}$, Soong et al. (10) y Templeman ${ }^{(11)}$ han publicado sus resultados en la creación de neovagina asistida por laparoscopía usando peritoneo pélvico. El peritoneo que se utiliza como tejido de recubrimiento de la neovagina permite el desarrollo de un $\mathrm{pH}$ vaginal ácido y es reemplazado por epitelio escamoso seis meses después de la cirugía ${ }^{(9)}$.

En comparación con la técnica convencional de McIndoe y otras opciones quirúrgicas, la creación laparoscópica de neovagina utilizando peritoneo movilizado tiene numerosas ventajas. En primer lugar, el procedimiento no ocasiona cicatrices externas, como aquellas asociadas con la técnica de injerto de 
piel. En segundo lugar, la técnica es simple en manos experimentadas y tiene los beneficios de la cirugía mínimamente invasiva con rápida recuperación, menor dolor post operatorio, rápida recuperación y mínimos cuidados a corto y largo plazo, y no requiere laparotomía. En tercer lugar, el procedimiento no se asocia con dispareunia o tejido de granulación en neovagina, observados con frecuencia con otras técnicas, obteniéndose mejores beneficios cosméticos y económicos.

Es importante mencionar que se obtiene una adecuada longitud de vagina, sin tendencia a la estenosis, lo cual redunda en buenos resultados en las relaciones coitales ${ }^{(12)}$. A diferencia de Soong et al., quienes emplearon molde vaginal rígido en el período post operatorio ${ }^{(10)}$, o Templeman ${ }^{(1)}$ y Fedele ${ }^{(7)}$ que utilizaron molde de látex blando inflable o lavable, respectivamente, nosotros preferimos utilizar molde vaginal blando descartable hecho con espuma de goma y cubierto con condón, el que es usado solamente al acostarse, lo que causa menores molestias a la paciente. En 2013 Altez et al., presentaron el caso de una paciente operada con esta técnica, con buenos resultados ${ }^{(13)}$; nosotros estamos presentando la primera serie de casos de creación de neovagina asistida por laparoscopía utilizando revestimiento peritoneal, en nuestro país y en la región.

La creación de una neovagina empleando peritoneo pélvico con un abordaje asistido por laparoscopía, seguido de un molde vaginal blando de espuma de goma, es una técnica eficiente y segura, que permite un alta precoz con mínimas molestias post operatorias, y provee a las pacientes de una vagina funcional, que les permite tener una adecuada vida sexual.

\section{Declaración de conflictos de interés:}

El autor declara no tener conflictos de interés en la publicación de este reporte de casos.

\section{Correspondencia:}

Adolfo Rechkemmer Prieto

Clínica Miraflores

JA Encinas 141, Miraflores, Lima 1, Perú.

Correo electrónico: adolfo.rechkemmer@upch.pe

\section{REFERENCIAS BIBLIOGRÁFICAS}

1. Dietrich JE. Surgical management of reproductive tract anomalies. En: Handa V, Van-Le L. Te Linde"s Operative Gynecology. New York, Ed. Wolters Kluwer; 2020. p. 1131-1160.

2. Frank RT. The formation of an artificial vagina without operation. Am J Obste Gynecol 1938; 35:1053-5. doi: 10.1016/S0002-9378(38)90400-4

3. McIndoe AH, Bannister JB. An operation for cure of congenital absence of the vagina. J Obstet Gynaecol Br Emp 1938; 45:490-4

4. Pratt JH. Vaginal atresia corrected by use of small and large bowel. Clin Obstet Gynecol 1972; 15:639.

5. McCraw JB, Massey FM, Shanklin KD, et al. Vaginal reconstruction with gracilis myocutaneous flaps. Plast Reconstr Surg. 1976; 58:176.

6. Vecchietti G. Neovagina nella syndrome di Rokitansky-Kuster-Hauser. Attual Ostet Ginecol. 1965; 11:131-47.

7. Fedele L, Busacca M, Candiani M, Vignali M. Laparoscopic creation of a neovagina in MayerRokitansky-Kuster-Hauser syndrome by modification of Vecchietti's operation. Am J Obstet Gynecol. 1994; 171:268-9.

8. Davydov SN. Colpopoiesis from peritoneum of the uterorectal space. Obstet Gynecol (Moscow). 1969; 12: 55-57.

9. Adamyan LV. Additional international perpectives. En: Nichols DH. Gynecologic and Obstetric Surgery. New York: Ed Mosby; 1993. p. 1167-82.

10. Soong YK, Chang FH, Lai YM, et al. Results of modified laparoscopically assisted neovaginoplasty in 18 patients with congenital absence of vagina. Hum Reprod. 1996; 11:200-213.

11. Templeman CL, Hertweck SP, Levine RL, Reich H. Use of laparoscopically mobilized peritoneum in the creation of a neovagina. Fertil Steril. 2000; 74:58992.

12. Giannesi A, Marchiole P, Benchaib M, ChevretMeasson M, Mathevet P, Dargent D. Sexuality after laparoscopic Davidov in patients affected by congenital complete vaginal agenesis associated with uterine agenesis or hypoplasia. Hum Reprod. 2005; 20 (10):2954-2957.

13. Altez C, Molina E, Ortega F, Angulo D. Neovagina con revestimiento peritoneal por laparoscopía. Rev Per Ginecol Obstet. 2013; 1:55-57.

Recibido: 20/04/2020

Aceptado: 28/12/2020 